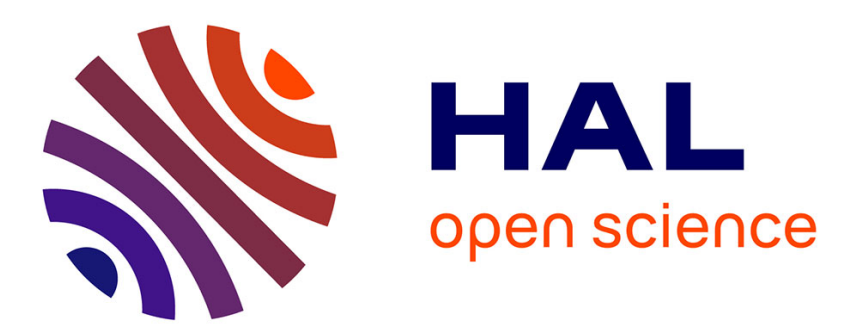

\title{
Characterization and Comparison of Rover Locomotion Performance Based on Kinematic Aspects
}

\author{
Thomas Thueer, Roland Siegwart
}

\section{To cite this version:}

Thomas Thueer, Roland Siegwart. Characterization and Comparison of Rover Locomotion Performance Based on Kinematic Aspects. 6th International Conference on Field and Service Robotics FSR 2007, Jul 2007, Chamonix, France. inria-00202691

\section{HAL Id: inria-00202691 https://hal.inria.fr/inria-00202691}

Submitted on 7 Jan 2008

HAL is a multi-disciplinary open access archive for the deposit and dissemination of scientific research documents, whether they are published or not. The documents may come from teaching and research institutions in France or abroad, or from public or private research centers.
L'archive ouverte pluridisciplinaire HAL, est destinée au dépôt et à la diffusion de documents scientifiques de niveau recherche, publiés ou non, émanant des établissements d'enseignement et de recherche français ou étrangers, des laboratoires publics ou privés. 


\title{
Characterization and Comparison of Rover Locomotion Performance Based on Kinematic Aspects
}

\author{
Thomas Thueer and Roland Siegwart \\ Autonomous Systems Lab, ETH Zurich, 8092 Zurich, Switzerland \\ \{thomas.thueer, roland.siegwart\}@mavt.ethz.ch
}

Summary. Evaluation and comparison of locomotion performance of rovers is a difficult, though very important issue. The performance is influenced by a large number of parameters. In this work, three different rovers were analyzed from a kinematic point of view. Based on a kinematic model, the optimal velocities at the actual position were calculated for all wheels and used for characterization of the suspension of the different rovers. Simulation results show significant differences between the rovers and thus, the utility of the chosen metric. It is shown that a substantial reduction of slip can be achieved by integrating kinematics in a modelbased velocity controller.

\section{Introduction}

Many rovers have been presented in the past by different people and institutions, however, comparison of performance was widely neglected and the advantages of new systems were highlighted without an absolute reference. It is difficult to compare systems that differ in all dimensions, e.g. wheel diameter, footprint, centre of gravity (CoG), and which were developed with respect to different requirements but comparison can yield important information about the systems' real capabilities.

An effort in this direction was made at the Autonomous Systems Lab (ASL) in order to compare a selection of wheeled, passive systems based on precisely defined metrics. The first steps towards a complete evaluation of these systems included the development of a quasi-static simulation tool [1], an analysis of a wide selection of locomotion systems [2] and a validation of the simulation results on real breadboards of selected rovers [3].

This paper describes the continuation of the rover comparison based on another aspect: kinematics. Several people have presented work that focuses on kinematics for different purposes. Forward kinematics was used in simulation for the estimation of rover position and heading in [4]. Wheel actuation commands can be derived for a desired rover motion by means of an inverse 


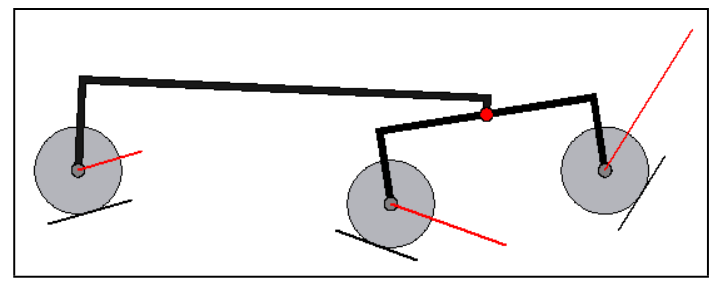

Fig. 1. Example of optimal velocity distribution depending on rover state.

kinematics model [5]. [6] and [7] included rover kinematics in the estimation of the wheel-ground contact angle and [8] developed a kinematic observer for articulated rovers.

A kinematic model can provide valuable information because the ability of articulated rovers to adapt to uneven terrain makes it difficult to relate rover motion to wheel motion and requires the wheels to move at different speeds. In this context rover control plays a central role. Several control strategies have been presented which aim at increasing the rover's performance by synchronizing wheel velocities [9], setting optimal torques depending on the rover's state [10] or actively adapting the rover's configuration based on kinematic information [11].

This work's main focus lies on the development of a metric that helps characterizing the kinematic potential of different suspension types. Kinematics are used to calculate the optimal velocity distribution depending on the rover's state in rough terrain. Fig. 1 shows that optimal velocities on the wheels differ significantly if kinematic constraints of the suspension have to be respected. The bigger the difference between optimal velocities, the more difficult it is to satisfy the constraints. Violation of such constraints induces slip. Therefore, the different rovers will be analyzed with regards to the velocity distribution that is caused by their suspension mechanism and the resulting slip. Additionally, the kinematic model is integrated into a control loop which adapts the velocity commands for each wheel separately in order to reduce slip.

The next section describes how the kinematic models were derived for the different rovers. Then the metrics are introduced which allow to properly compare locomotion performance. Further, the simulation setup is presented in which the tests were performed before the results are discussed. Finally, the presented work is summarized.

\section{Kinematic models of rovers}

In this section the analyzed rovers are briefly presented and the kinematic modeling is explained. For reasons of consistency, the same rovers were selected for the kinematic analysis as in [3] where more information can be found about the systems. The selected rovers are shown in Table 1 . The kinematic 
models were simplified such that they still respect the geometrical constraints imposed by the suspension system, thus maintaining identical behavior as the real rovers. Since the original rovers differ with respect to many parameters, they were all normalized to the approximate dimensions of the Mars Exploration Rovers ${ }^{1}$ (MER) [12] with the same total mass ( 180kg), wheels $(\varnothing 0.25 \mathrm{~m})$, foot print $(\sim 1.5 \mathrm{~m})$ and CoG. This normalization allows for a proper comparison of the suspension types regardless of terrain characteristics.

Rough-terrain robots usually consist of several rigid elements connected through joints of a certain number of degrees of freedom (DoF) resulting in a structure that has one system DoF. This allows the rovers to move along an uneven terrain without loosing contact (in most cases). Rigid body kinematics for closed loop systems was used to represent these characteristics and establish the rover models. The modeling process is explained on the example of the MER as it is depicted in Fig. 2.

\footnotetext{
${ }^{1}$ Nominal driving direction of MER (trailing bogie) in opposite direction to definition in Table 1.
}

Table 1. Selected rovers: MER, CRAB and RCL-E.

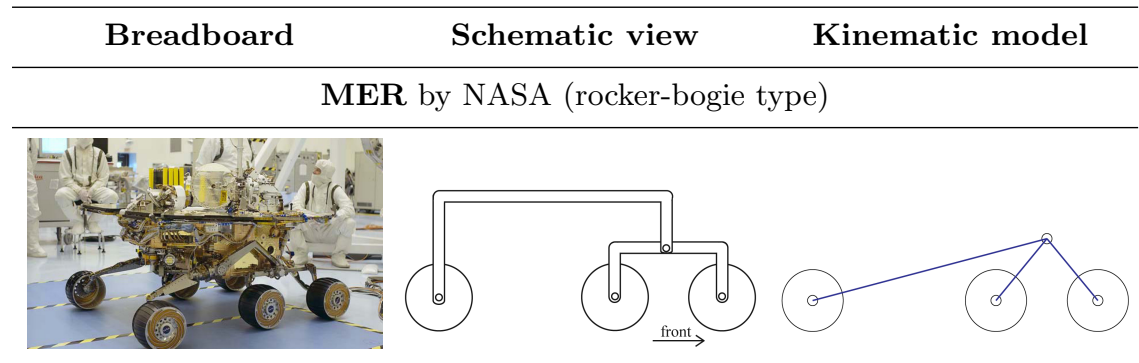

CRAB by ASL (symmetric structure based on four parallel bogies)

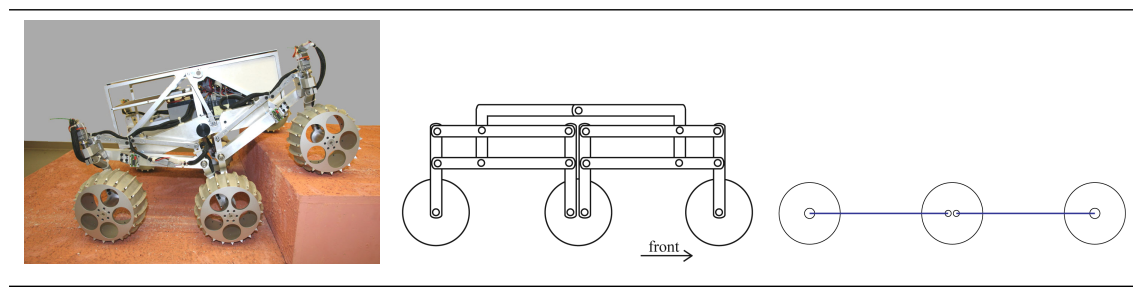

RCL-E by RCL (three parallel bogies, no differential mechanism)

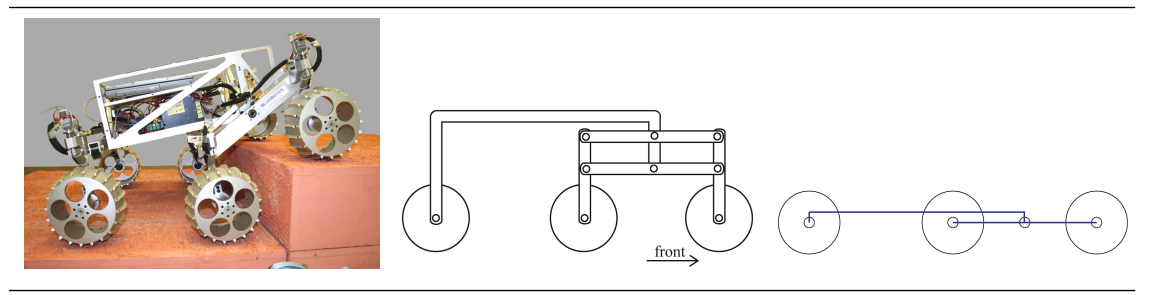




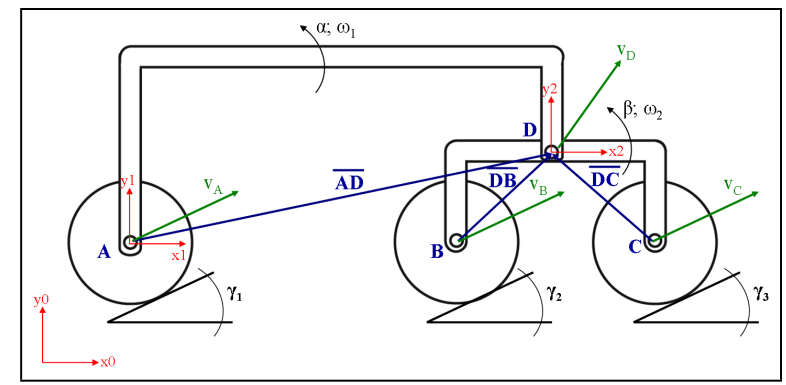

Fig. 2. Kinematic model of MER with parameter definition.

The system is defined through the vectors of constant length $\mathrm{AD}, \mathrm{DB}$ and DC, wheel-ground contact angles $\left(\gamma_{i}\right)$ as well as orientation of rocker $(\alpha)$ and bogie $(\beta)$ with respect to the inertial system. In reality $\alpha$ could be measured by means of an inertial measurement unit (IMU) and $\beta$ with an angular sensor in $\mathrm{D}$ that measures the relative movement between rocker and bogie. Measuring the wheel-ground contact angles requires tactile wheels as in [13]. The development of tactile wheels is an ongoing project at ASL.

The unknown parameters are the magnitudes of the velocities in A, B, C and $\mathrm{D}$ (given that the wheels always touch the ground), as well as the rotational velocities of rocker $\left(\omega_{1}\right)$ and bogie $\left(\omega_{2}\right)$. The velocity in D is not of interest; therefore it is expressed by means of the other velocities yielding an equation system with four equations (2D) and five unknowns. As it was mentioned before rovers have one DoF, which means that one velocity can be chosen as input. Thus, the equation system has exactly one solution. $\vartheta_{D}$ is expressed in the inertial system through A, B and C.

$$
\begin{aligned}
& \vartheta_{D_{A}}:=\vartheta_{D}=\vartheta_{A}+\omega_{1} \times{ }_{1}^{0} R(\alpha){ }^{1} \overline{A D} \\
& \vartheta_{D_{B}}:=\vartheta_{D}=\vartheta_{B}+\omega_{2} \times{ }_{2}^{0} R(\beta){ }^{2} \overline{D B} \\
& \vartheta_{D_{C}}:=\vartheta_{D}=\vartheta_{C}+\omega_{2} \times{ }_{2}^{0} R(\beta)^{2} \overline{D C}
\end{aligned}
$$

where $\vartheta_{i} \quad=$ velocity in $i$ w.r.t. inertial system,

$\omega_{i}=$ rotational velocity of system $i$ w.r.t. inertial system,

${ }_{j}^{i} R(m)=$ transformation from system $j$ to $i$ by rotation of angle $m$,

${ }^{i} \overline{X Y}=$ vector from $X$ to $Y$ expressed in coordinate system $i$.

The equation system becomes

$$
\vartheta_{D_{A}}=\vartheta_{D_{B}} ; \quad \vartheta_{D_{A}}=\vartheta_{D_{C}}
$$

and can be written as

$$
A x=b \quad \text { with } \quad x=\left(\vartheta_{B}, \vartheta_{C}, \omega_{1}, \omega_{2}\right)^{T}
$$




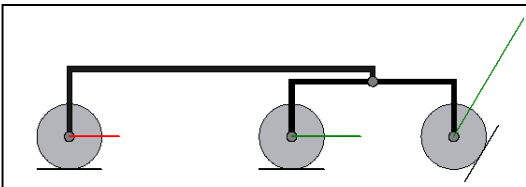

(a) Reference on rear wheel

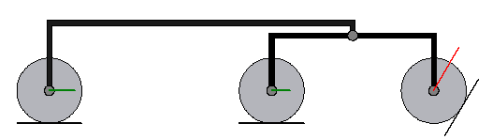

(b) Reference on front wheel

Fig. 3. Velocity distribution with different reference wheels

Equation 5 can be easily solved for $x$ with $\vartheta_{A}$ as input. If the velocity input is given at $\mathrm{B}$ or $\mathrm{C}$ the system has to be modified such that $\vartheta_{B}$ or $\vartheta_{C}$ appear in $b$. Models of the same form were also generated for CRAB and RCL-E. Since the kinematic model takes only one input velocity special attention has to be paid to the selection of the reference where this input is set. This problem of reference selection needs extended explanation.

In Fig. 3 the MER is depicted twice in the same position but with different reference wheels. The velocity of the reference wheel is the same for both cases (red line). The green lines indicate the corresponding optimal velocities on the other wheels. Given, the rover is on a flat surface and encounters a step-like obstacle, either the front wheel has to speed up the obstacle (Fig. 3(a)) or the rear wheel has to slow down significantly (Fig. 3(b)) in order to satisfy the kinematic constraints. In the first case, the front wheel is very likely to slip because it cannot accelerate sufficiently fast. In the second case, slip may be avoided on the front wheel, but the global velocity of the rover is considerably reduced. This kind of problem is inherent to the articulated structure of the suspension system. It is very important to treat this issue of reference selection carefully because it can influence the performance of the system.

\section{Metrics}

It is important to have metrics that precisely define what is considered a good or bad performance. The metrics used in this study are described below.

\subsection{Difference between input and optimal velocities: $\Delta v e l_{o p t}$}

This metric is a measure for the risk of violation of kinematic constraints through deviation from optimal velocity. This is the case, e.g., if a simple velocity control is used which sets equal speed on all wheels.

$$
\Delta v e l_{o p t}=\sum_{i=1}^{n}\left|\vartheta_{\text {ref }}-\vartheta_{\text {opt }_{i}}\right| \quad \text { with } \quad i \neq \text { ref }
$$

where $\vartheta_{\text {ref }}=$ velocity of reference wheel,

$\vartheta_{\text {opt }_{i}}=$ optimal velocity of wheel $i$,

$n \quad=$ number of wheels. 
As it was mentioned above, uneven terrain leads to different optimal velocities on the wheels. If the controller is not able to set the velocities accordingly, slip occurs because kinematic constraints are broken. $\Delta v e l_{\text {opt }}$ is an indicator how good a suspension system adapts to the terrain while respecting kinematic constraints and not needing a sophisticated controller. If this value is small it is easier to control the rover because the kinematics impose similar wheel velocities, and the risk of slip is smaller.

\subsection{Slip}

Slip is defined as the difference between the displacement of a wheel measured at the wheel center point and the displacement derived from wheel rotation measurement with encoders.

$$
\text { slip }=\sum_{i=1}^{n} \mid \Delta \text { pos }_{\text {wheelcenter }_{i}}-\Delta \text { pos }_{\text {encoder }_{i}} \mid
$$

Slip is bad for the odometry and it is a waste of energy because it does not contribute to the movement of the rover. Therefore this value must be small for good performance.

\section{Simulation setup}

All simulations were performed using the software Working Model 2D (WM2D) by Design-Simulation [14]. It provides a nice graphical user interface (GUI) for easy definition of simulation runs. Fig. 4 depicts a normalized rover model on an randomly generated, uneven terrain. WM2D has an integrated interface to exchange data with Matlab in which the kinematic model is implemented (Fig. 5). The motors are velocity controlled, i.e., constant velocity input.

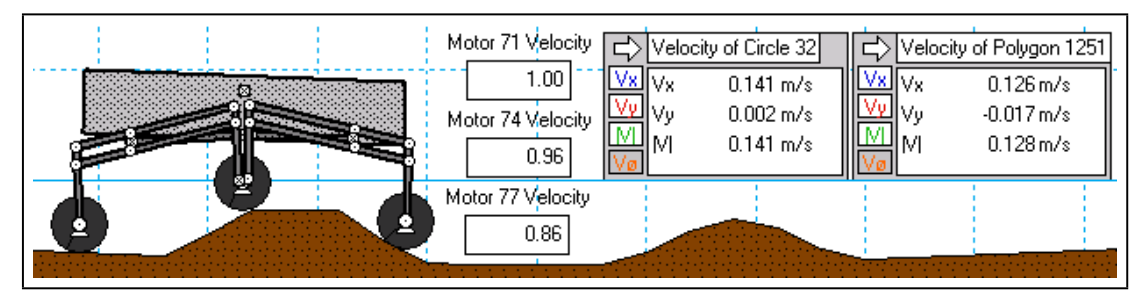

Fig. 4. Graphical user interface WM2D with CRAB. 


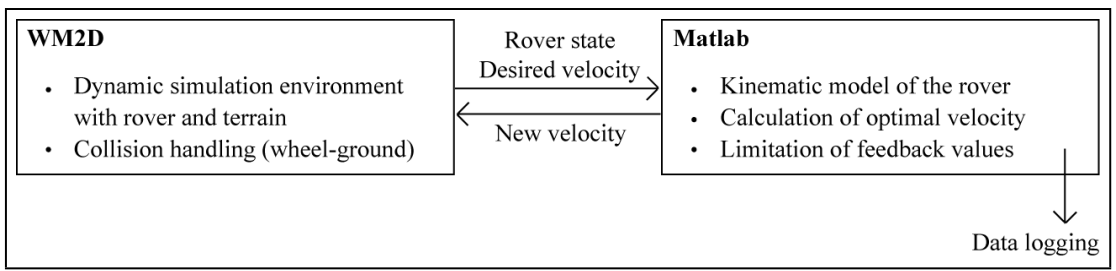

Fig. 5. Interaction of WM2D and Matlab.

\section{Simulation results}

The same simulations were performed for all three rovers under equal conditions. The results are presented in this section. First, the parameters that define a simulation are described.

\section{- Terrain}

The selection of the terrains was motivated by the idea to have two distinct types: uneven terrain to represent a real environment ( $24 \mathrm{~m}$ long, sinusoidal obstacles with defined max. height); artificial benchmark obstacle that allows analyzing what happens during obstacle negotiation due to decoupled climbing phases of the wheels (truncated pyramid, height equal to wheel diameter).

\section{- Control}

First, the rovers were assessed with constant speed control, then the optimal velocity information was added to the controller (model-based).

\section{- Reference wheel}

Indicates the wheel used as reference (1: rear; 2 : middle; 3 : front).

Table 2 lists the results for the metric $\Delta v e l_{\text {opt }}$. The values correspond to the sum of $\Delta v$ el $_{\text {opt }}$ over the whole simulation run. For these tests constant speed control was used in combination with the artificial obstacle (tests 1-3) and uneven terrain (tests 7-9).

Tests 1-3 show that there is a significant difference between the performance of MER $(\sim 15-27 \mathrm{~m} / \mathrm{s})$ and the other rovers $(\sim 10-13 \mathrm{~m} / \mathrm{s})$, CRAB and RCL-E. This means that, if a constant speed control was used on the rovers, the error would be much bigger on MER or, in other words, MER has a higher need to adapt the wheel velocities in order to satisfy the kinematic constraints and reduce slip. The performance is influenced by the selection of the reference wheel for all rovers, whereas the results for MER show the biggest influence, i.e., the performance varies by almost $50 \%$. It is interesting that the performance of CRAB reflects the symmetrical suspension with same results for front and rear wheel. The results from tests 1-3 are confirmed by tests 7-9 on uneven terrain.

Table 3 shows the performances regarding the slip metric on two different terrains, as well as with constant speed and model-based control. Note that 
Table 2. Simulation results for metric $\Delta v e l_{\text {opt }}$.

\begin{tabular}{|c|c|c|c|c|c|}
\hline Rover & $\begin{array}{c}\text { Ref. } \\
\text { wheel }\end{array}$ & Test & $\sum_{[\mathrm{m} / \mathrm{s}]}\left(\Delta v e l_{o p t}\right)$ & Test & $\sum\left(\Delta v e l_{\text {opt }}\right)$ \\
\hline MER & \multirow{3}{*}{1} & \multirow{3}{*}{1} & 27.87 & \multirow{3}{*}{7} & 93.71 \\
\hline CRAB & & & 12.17 & & 37.53 \\
\hline RCL-E & & & 12.72 & & 35.69 \\
\hline MER & \multirow{3}{*}{2} & \multirow{3}{*}{2} & 15.87 & \multirow{3}{*}{8} & 55.46 \\
\hline CRAB & & & 10.00 & & 28.00 \\
\hline RCL-E & & & 11.12 & & 29.96 \\
\hline MER & \multirow{3}{*}{3} & \multirow{3}{*}{3} & 17.25 & \multirow{3}{*}{9} & 55.20 \\
\hline CRAB & & & 12.02 & & 37.87 \\
\hline RCL-E & & & 11.69 & & 33.70 \\
\hline \multicolumn{2}{|c|}{ Terrain type } & \multicolumn{2}{|c|}{ Truncated pyramid } & \multicolumn{2}{|c|}{ Uneven terrain $24 \mathrm{~m}$} \\
\hline
\end{tabular}

Table 3. Simulation results for metric slip.

\begin{tabular}{|c|c|c|c|c|c|c|c|}
\hline \multirow[t]{2}{*}{ Rover } & \multirow{2}{*}{$\begin{array}{c}\text { Ref. } \\
\text { wheel }\end{array}$} & \multirow[t]{2}{*}{ Test } & \multicolumn{2}{|c|}{ Slip (control type) } & \multirow[t]{2}{*}{ Test } & \multicolumn{2}{|c|}{ Slip (control type) } \\
\hline & & & const.speed & model-based & & const.speed & model-based \\
\hline MER & \multirow{3}{*}{1} & \multirow{3}{*}{$1 / 4$} & 1.12 & 0.61 & \multirow{3}{*}{$7 / 10$} & 4.19 & 2.46 \\
\hline CRAB & & & 0.64 & 0.24 & & 1.89 & 1.00 \\
\hline RCL-E & & & 0.69 & 0.21 & & 1.97 & 0.94 \\
\hline MER & \multirow{3}{*}{2} & \multirow{3}{*}{$2 / 5$} & 1.12 & 0.49 & \multirow{3}{*}{$8 / 11$} & 4.19 & 1.99 \\
\hline CRAB & & & 0.64 & 0.20 & & 1.89 & 0.85 \\
\hline RCL-E & & & 0.69 & 0.22 & & 1.97 & 1.23 \\
\hline MER & \multirow{3}{*}{3} & \multirow{3}{*}{$3 / 6$} & 1.12 & 0.51 & \multirow{3}{*}{$9 / 12$} & 4.19 & 1.94 \\
\hline CRAB & & & 0.64 & 0.22 & & 1.89 & 1.02 \\
\hline RCL-E & & & 0.69 & 0.22 & & 1.97 & 0.99 \\
\hline \multicolumn{2}{|c|}{ Terrain type } & \multicolumn{3}{|c|}{ Truncated pyramid } & \multicolumn{3}{|c|}{ Uneven terrain $24 \mathrm{~m}$} \\
\hline
\end{tabular}

the selection of the reference wheel does not influence the results in constant speed mode because all wheels have the same input velocity. CRAB and RCL-E show similarly good performance while slip for MER is substantially bigger. This ranking is the same for all simulation types whether the rovers move on uneven terrain or have to cross the truncated pyramid.

As it was to be expected, a significant improvement can be observed for all rovers if the model-based control is activated, i.e., the input velocities are adapted according to the optimal velocities from the kinematic model. The performance can be improved by up to $70 \%$. However, considerable differences in performance, and thus the ranking, remain.

Slip and optimal velocity of all rovers for test 7 are depicted in Fig. 6. The graphs illustrate the results discussed above: similar slip values of CRAB and 


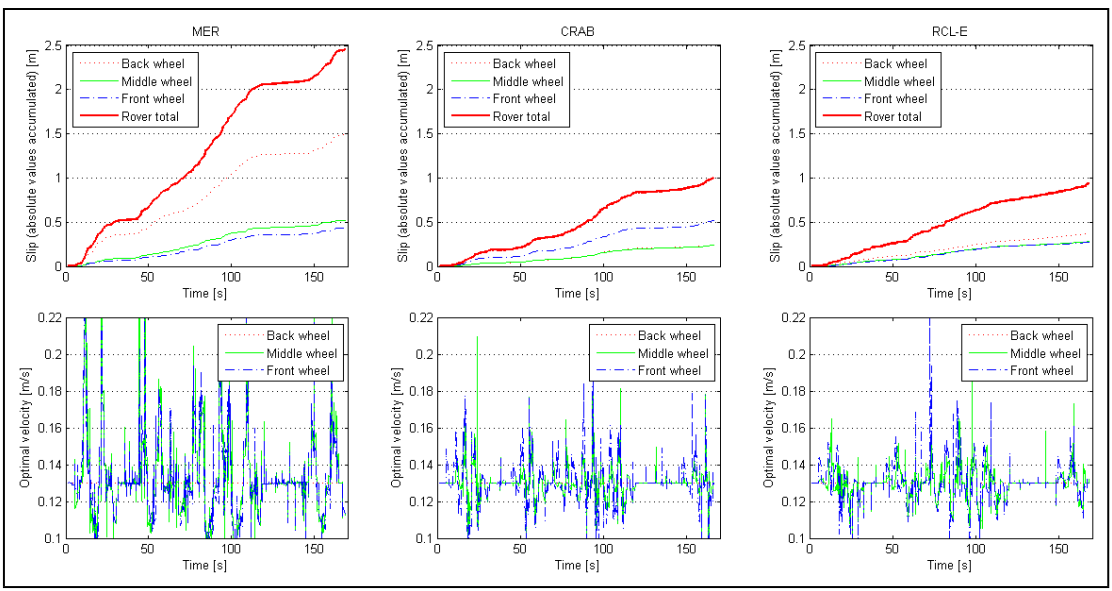

Fig. 6. Slip and optimal velocity of all rovers with rear wheel as reference.

RCL-E; more than twice the final value of total slip of MER. The deviation of optimal velocity from input velocity $(0.13[\mathrm{~m} / \mathrm{s}])$ is the biggest for MER.

\section{Conclusion and Future Work}

Rover locomotion performance was analyzed from a kinematic point of view. The main focus was laid on characterization of suspension systems. Additionally to a simple analysis three different systems were compared based on two different metrics. The new metric $\Delta v e l_{\text {opt }}$ was introduced with the aim to characterize the suspension systems in terms of compliance with kinematic constraints while moving on uneven terrain. For this, the optimal wheel velocities depending on the rover's state were calculated by means of a kinematic model. Slip was used as a second metric because it occurs if kinematic constraints are violated. A large number of simulations was performed with different settings. The rovers CRAB and RCL-E performed well with respect to both presented metrics while MER's performance was significantly inferior. It was also shown how integration of kinematics in a model-based controller improves performance.

In future work motor dynamics will be added to the simulation environment and kinematic investigations will be extended.

\section{References}

1. A. Krebs, T. Thueer, S. Michaud, and R. Siegwart. Performance optimization for passive rover structure: A 2d quasi-static tool. In IEEE International Conference on Intelligent Robots and Systems (IROS'06), Beijing, China, 2006. 
2. T. Thueer, A. Krebs, and R. Siegwart. Comprehensive locomotion performance evaluation of all-terrain robots. In IEEE International Conference on Intelligent Robots and Systems (IROS'06), Beijing, China, 2006.

3. T. Thueer, A. Krebs, P. Lamon, and R. Siegwart. Performance comparison of rough-terrain robots - simulation and hardware. International Journal of Field Robotics Vol.24 Issue 3 (Special Issue on Space Robotics Part I), Wiley, 2007.

4. Herv Hacot, Steven Dubowsky, and Philippe Bidaud. Analysis and simulation of a rocker-bogie exploration rover. In CISM-IFToMM Symposium, Paris, France, 1998.

5. M. Tarokh, G. McDermott, S. Hayati, and J. Hung. Kinematic modeling of a high mobility mars rover. In IEEE International Conference on Robotics and Automation (ICRA'99), volume 2, pages 992-998 vol.2, Detroit, Michigan, USA, 1999.

6. K. Iagnemma and S. Dubowsky. Vehicle wheel-ground contact angle estimation: with application to mobile robot traction control. In International Symposium on Advances in Robot Kinematics (ARK 00), 2000.

7. T. Peynot and S. Lacroix. Enhanced locomotion control for a planetary rover. In IEEE Internation Conference on Intelligent Robots and Systems (IROS'O3), volume 1, pages 311-316 vol.1, Las Vegas, USA, 2003.

8. J. Balaram. Kinematic state estimation for a mars rover. Robotica, 18:12, 2000.

9. E. T. Baumgartner, H. Aghazarian, and A. Trebi-Ollennu. Rover localization results for the fido rover. In G. T. McKee and P. S. Schenker, editors, SPIE Proc. Vol. 4571, Sensor Fusion and Decentralized Control in Autonomous Robotic Systems IV, Newton, MA, USA, 2001. wheel synchronisation control algorithm.

10. Pierre Lamon and Roland Siegwart. Wheel torque control in rough terrain modeling and simulation. In IEEE International Conference on Robotics and Automation (ICRA'05), Barcelona, Spain, 2005.

11. Christophe Grand, Faiz Ben Amar, and Philippe Bidaud. Kinematic analysis and stability optimization of a reconfigurable legged-wheeled mini-rover. In $U n$ manned Ground Vehicle Technology IV, volume 4715, pages 295-302, Orlando, FL, USA, 2002. SPIE.

12. R. A. Lindemann and C. J. Voorhees. Mars exploration rover mobility assembly design, test and performance. In IEEE International Conference on Systems, Man and Cybernetics, 2005, volume 1, pages 450-455, Waikoloa, Hawaii, USA, 2005.

13. M. Lauria, Y. Piguet, and R. Siegwart. Octopus - an autonomous wheeled climbing robot. In Fifth International Conference on Climbing and Walking Robots, Bury St Edmunds and London, UK, 2002. Professional Engineering Publishing Limited.

14. Design-Simulation. Working model 2d, http://www.designsimulation.com/wm2d/, 2006. 